\title{
st \\ Pensamento racional e criação científica em Poincaré
}

Michel PATY

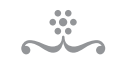

RESUMO

Neste artigo, esclarecemos o "estilo" de Henry Poincaré na física teórica e na matemática, considerando o caso de sua formulação teórica da eletrodinâmica relativista, e do lugar que nela tem o princípio de relatividade. A comparação desse trabalho criador com aquele paralelo e contemporâneo de Einstein (da relatividade especial) permite pôr em evidência os contrastes entre as duas abordagens. Tratamos, em seguida, da obra e do pensamento de Poincaré no campo da filosofia a respeito do problema da criação científica e de sua relação com a racionalidade.

PaLAVRas-Ghave • Criação científica. Intuição. Invenção. Racionalidade. Princípio de relatividade. Eletrodinâmica. Física teórica. Matemática.

\section{INTRODUÇÃO}

Apresentarei, a partir do pensamento e da obra de Henri Poincaré, o problema da relação entre a racionalidade e o pensamento criativo na matemática e na física matemática e teórica. Tratarei inicialmente de seu trabalho na física matemática e teórica, detendo-me em seu pensamento sobre a eletrodinâmica e o princípio de relatividade. Abordarei, em seguida, o aspecto reflexivo do pensamento de Poincaré sobre o tema da criação científica, que faz que ele seja um dos primeiros a preocupar-se com uma filosofia da criação segundo a racionalidade.

\section{RaGionalidade E PENSAMENTO GRIATIVO \\ NA MATEMÁTICA E NA FÍSICA MATEMÁTICA E TEÓRICA}

Tomadas separadamente, as duas expressões "pensamento racional" ou "racionalidade", de uma parte, e "criação científica", de outra parte, serão aceitas sem dificuldade, senão em geral, pelo menos no que diz respeito à obra de Henri Poincaré. 0 pensamento racional está evidentemente implicado na pesquisa científica, especialmente 
nas matemáticas e na física. Que o trabalho de Poincaré seja, além disso, de criação matemática e físico-matemática é também uma afirmação que será pouca contestada, de modo que estamos dispostos em geral a saudar o aspecto criativo e criador de seu pensamento, especialmente o matemático.

Mas que a criação matemática ou científica em geral esteja diretamente ligada à racionalidade, a saber, que podemos falar de criação racional a propósito das ciências, é uma afirmação que está longe de ter unanimidade (cf. Paty, 1999b). Para muitos, e especialmente nas correntes dominantes da filosofia do conhecimento do século xx, do positivismo lógico à filosofia analítica, passando pelo racionalismo crítico popperiano, a parte criativa do pensamento científico encontra-se entre dois estados de pensamento (de conhecimento) caracterizáveis racionalmente, dos quais a filosofia analisa as proposições, a saber, o estado do conhecimento anterior e aquele do conhecimento atual. Mas o próprio momento criador escaparia definitivamente, por natureza, à análise racional e evidenciaria, portanto, o irracional, psicológico ou social. Basta lembrar a esse propósito as declarações explícitas de Karl Popper e a distinção de Hans Reichenbach entre contexto da descoberta e contexto da justificação, sendo este último o único apropriado, segundo ele, ao tratamento racional da filosofia, distinção que por muito tempo se impôs e que ainda hoje tem frequentemente autoridade (cf. Popper, 1959, 1972; Reichenbach, 1938).

Tratarei brevemente do pensamento filosófico de Poincaré, que parece inscrever-se antecipadamente contra essa concepção, uma vez que a visão de Poincaré está totalmente centrada na investigação científica, isto é, na atividade criativa do pensamento em vista dos problemas das ciências. Ficarei restrito, por enquanto, a sua obra propriamente científica nas matemáticas e na física matemática e teórica. Seus pares matemáticos da Academia Real da Suécia encontraram uma fórmula significativa para indicar a razão da premiação de seu trabalho sobre o problema dos três corpos, o qual revelou uma grande fecundidade nos desenvolvimentos contemporâneos do estudo dos sistemas dinâmicos. Eles declaram que Poincaré teria inaugurado uma "nova maneira de pensar" nas matemáticas e na física matemática, ao lançar as bases da abordagem dita qualitativa, que se interessa pelos tipos de comportamento dos sistemas dinâmicos e das soluções dos sistemas de equações que os caracterizam. Essa nova via de abordagem qualitativa e estrutural revelava mais sobre os sistemas dinâmicos (não-lineares) que as próprias soluções particulares, as quais estão sujeitas aos efeitos de amplificação arbitrária das variações das condições iniciais e, assim, finalmente imprevisíveis.

"Nova maneira de pensar": esta expressão designa, sem analisá-la explicitamente, a racionalidade (a maneira de pensar nas matemáticas e na física matemática), como o agente e o efeito da criação desses novos métodos e desses novos objetos matemáticos. 
Além desse reconhecimento oficial, algo implícito, de uma criação racional por outros especialistas nesse tema, poder-se-ia retomar a obra de Poincaré, analisando seu "estilo científico próprio" e caracterizar seus modos de racionalidade e de criatividade. O próprio Poincaré forneceu as indicações com muitos detalhes a propósito da sua invenção das funções fucsinas ou automorfas (cf. Poincaré, 1908b, 1908c; Hadamard, 1945). A intervenção, na narrativa que ele nos deixou, de considerações psicológicas não deve nos enganar: a questão é, sobretudo, fundamentalmente acerca da racionalidade. O próprio Poincaré via muito bem como o processo de invenção comporta fases sucessivas, alternando o trabalho consciente, voluntário e racional, do pensamento com as fases de pensamento semiconsciente, semi-inconsciente, que não são resolúveis analiticamente; mas é sempre o pensamento consciente e sua colocação de problemas (com suas formulações de natureza racional) que continua a guiar o movimento do pensamento, mesmo ao longo das fases inconscientes, em seu jogo com os símbolos que representam os conceitos. Sem poder recorrer às reminiscências dos momentos da invenção no sentido estrito de nosso autor, momentos do surgimento de uma ideia que jamais foi pensada antes e que nunca nos serão dados com os segredos de sua subjetividade, resta-nos as obras escritas, pelas quais poderemos tentar retraçar os caminhos da inteligibilidade seguindo a sua própria marca que é aquela de seu estilo de pensamento. E isso, tanto mais que, em Poincaré, a redação parece frequentemente reproduzir em sua espontaneidade o movimento do pensamento inventivo.

Podemos analisar desse mesmo modo suas contribuições na física e caracterizar, ainda aqui, seu estilo de pensamento e de trabalho, o qual esclarece sua maneira de compreender os problemas teóricos em relação aos fenômenos considerados. Certamente, o estilo de Poincaré na física é diferente de seu estilo nas matemáticas, devido à diferença dos objetos considerados (destaquemos a esse propósito que Poincaré também era físico propriamente falando, e que sua física matemática, como ele a chamava, também era, quanto ao objeto que ele havia proposto, uma física teórica (cf. Paty, 1999a). Contudo, se seu estilo na física difere de seu estilo nas matemáticas, os dois estão mutuamente relacionados. Acontece-lhe de nutrir sua intuição matemática com o pensamento de situações físicas; ele faz geralmente apelo, no exercício de seu pensamento físico, aos elementos que provêm de seu pensamento matemático e que lhe permitem ligar imediatamente situações de aparências diferentes, mas estruturalmente semelhantes (seria necessário lembrar aqui sua concepção de "analogias matemáticas"). Ou ainda, ele chega a pensar em termos de grupos, como em seu trabalho sobre a eletrodinâmica relativista finalizado em 1905, mas publicado em 1906, sobre as transformações de referenciais pelas quais ele define o grupo de Lorentz. Esse pensamento acerca de grupos corresponde de forma eminente a uma visão sintética, que lhe permitia ter 
diretamente acesso às propriedades características dos sistemas físicos considerados (da adição relativista das velocidades às invariantes das transformações de Lorentz).

Suas contribuições para a física tomam geralmente como ponto de partida um estudo crítico das teorias existentes e de sua relação com os dados da observação ou da experiência. Esse estudo crítico é um levantamento racional dos problemas abordados, que são assim confrontados com as exigências da inteligibilidade. O "hábito matemático" facilita a percepção sintética para o entendimento dos elementos do problema, e Poincaré encontra-se, assim, frequentemente levado a propor vias originais para evidenciar de plano a dificuldade de certa teoria em vigor e para indicar as possibilidades de aperfeiçoamento ou de modificações ou, às vezes, simplesmente para revelar os limites que lhe são inerentes. Por exemplo, acerca da natureza da luz, que se manifesta por traços de fenômenos sobre os quais a "teoria matemática da luz", inaugurada por Fresnel, permanece silenciosa.

Consideremos esse caso mais detalhadamente. A fórmula de Fresnel "sobre o arrastamento parcial do éter", acrescentada a sua teoria das ondas luminosas, para dar conta das aberrações das estrelas e da experiência de Arago acerca do prisma produzido pelo movimento da Terra, exprimia a modificação da velocidade da luz em um meio refringente quando este está em movimento. Essa modificação permitia manter inalterada a lei da refração no prisma em vigor, quando o prisma está em repouso em relação ao éter, suposto suporte das ondas luminosas. O "coeficiente de Fresnel” dessa fórmula' (que intervém "em primeira ordem em $v / c$ ", v e $c$ sendo, respectivamente, a velocidade de arrastamento da Terra em seu movimento anual e a velocidade da luz no vazio) compensava, por assim dizer, os efeitos do movimento sobre as leis da óptica. A hipótese para a qual Fresnel tinha formulado seu coeficiente estava baseada em um modelo do éter (em termos de densidade de moléculas do meio etéreo, suporte das ondas luminosas). No caso da dupla refração, estudada por Eleuthère Mascart, onde dois raios refratados são produzidos por um raio incidente de comprimento de onda dado, Mascart tinha encontrado que o coeficiente de arrastamento parcial do éter, combinado com a consideração do efeito Doppler-Fizeau sobre o comprimento de onda, é verificado para cada um dos raios desviados (o raio ordinário e o raio extraordinário), com seu comprimento de onda próprio e o índice correspondente. Ao contrário, no modelo do éter tal como Fresnel o formulava, a refração dos dois raios deveria fazer-se segundo o índice médio. Esse tratamento, resultante das experiências sistemáticas de Mascart, efetuadas entre 1872 e 1874 (cf. Mascart, 1872, 1874; Olivei-

1 A fórmula de Fresnel do arrastamento parcial é c'/n = c/n +- v/c $\left(1-1 / n^{2}\right)$, sendo $n$ o índice de refração do meio refringente (notação moderna). 
ra, 1993), foi determinante na escolha de Poincaré da teoria eletromagnética da luz proposta por Maxwell, na versão mais precisa de Hendryk Lorentz, com os elétrons vibrando como fontes do campo eletromagnético e um éter imóvel (cf. Maxwell, 1954 [1873]; Lorentz, 1895).

Para Poincaré, esse caráter revelava, por um lado, uma limitação inerente a uma teoria puramente matemática da luz e indicava que era preciso chegar a uma teoria que se pronunciasse sobre a natureza física da irradiação luminosa; por outro lado, entre as teorias físicas da luz, aquela de Maxwell-Lorentz obtinha como consequência, sem modelo particular de éter, o coeficiente de Fresnel para cada comprimento de onda. A experiência de Fizeau (realizada em 1851), que materializava, por assim dizer, o coeficiente de Fresnel, e o resultado de Mascart, que tornava precisa a dependência deste último em função do comprimento de onda dos raios, como acabamos de recordar, conduziram, portanto, Poincaré a escolher a teoria de Maxwell-Lorentz, de preferência às outras teorias concorrentes, especialmente a de Maxwell-Hertz de um éter luminoso eletromagnético totalmente arrastado pelo movimento dos corpos em sua vizinhança. Ele diagnosticou, contudo, as insuficiências na formulação dessa teoria, que anunciou em 1895 e que não parou de aperfeiçoar em seguida até chegar em 1905 a seu resultado, paralelo àquele de Einstein.

O caminho próprio de Poincaré nesse assunto, diferente do de Einstein (os dois obtiveram, entretanto, resultados convergentes, mas com significação estrutural diferente), foi o de fazer da teoria eletromagnética - uma teoria da "física matemática" em um sentido tão pleno como a mecânica analítica lagrangiana - um modelo ou ideal comum aos físicos teóricos da época (sem "redução mecanicista", isto é, sem recorrer a modelos mecânicos particulares). Sobre esse problema teórico (obter uma maior racionalização da teoria eletromagnética), um estudo comparativo das respectivas contribuições de Lorentz, Poincaré e Einstein mostra a diferença entre "estilos científicos" e as exigências de inteligibilidade próprias a cada um desses três grandes pesquisadores, cujos resultados obtidos convergem para as relações de grandezas vizinhas, ou semelhantes, mas interpretadas diferentemente, isto é, correspondendo a uma compreensão conceitual diferente (cf. Paty, 1993, 1996).

\section{Lorentz, Poincaré e Einstein: eletrodinÂmica \\ E PRINCÍPIO DE RELATIVIDADE. ESTILO GIENTÍFIGO E GRIATIVO}

Lorentz e Poincaré, após uma dezena de anos de trabalho sobre essas questões, baseando-se na primeira teoria de Lorentz (1895), que mencionamos acima, abordaram o problema da eletrodinâmica dos corpos em movimento de uma maneira semi- 
empírica (cf. Lorentz, 1904; Poincaré, 1905b, 1905c). Eles visavam, primeiramente, o resultado negativo da experiência de Michelson-Morley sobre o vento do éter (experiência de segunda ordem em $v / c$ ), e a hipótese suplementar de Lorentz da contração dos comprimentos (nos braços do interferômetro) no sentido do movimento, generalizando-a em uma transformação de coordenadas que implicava também um "tempo local" para o sistema em movimento, diferente do "tempo físico" universal do sistema em repouso. Sem analisar, nesse trabalho de 1905 e publicado em 1906, o significado físico desse tempo local, que contradizia o tempo absoluto newtoniano, Poncaré o admitiu como físico, tal como o tempo que um relógio dá localmente, ao contrário de Lorentz, para quem não se tratava senão de uma transformação matemática auxiliar. ${ }^{2}$ As mesmas fórmulas de transformação tiveram como consequência uma lei da adição das velocidades diferente da adição galileana: essa propriedade, notada por Poincaré, não o foi por Lorentz (nos seus trabalhos de 1904, e 1905, respectivamente). Poincaré obteve assim a invariância da velocidade da luz e uma forma invariante das equações de Maxwell-Lorentz sob as transformações do referencial de Lorentz, o que implicava respeitar o princípio de relatividade. As equações invariantes da teoria eletromagnética de Lorentz corresponderam a uma eletrodinâmica relativista.

Poincaré foi igualmente mais longe que a teoria eletromagnética quando considerou o caráter finito da velocidade de propagação da luz, se essa velocidade fosse também a da gravitação, obrigaria a modificar a lei de Newton. Por meio dessa modificação, ele propôs uma abordagem totalmente nova e de longo alcance, que seria posteriormente seguida por todos os físicos que tinham que trabalhar com os grupos de transformação: pesquisar as grandezas que deixam a lagrangiana invariante. Para obter uma formulação matematicamente mais homogênea da relação entre as coordenadas do espaço e do tempo, dada na invariante $\mathrm{s}^{2}=\mathrm{x}^{2}-\mathrm{c}^{2} \cdot \mathrm{t}^{2}$, propôs escrever a variável tempo como uma quarta coordenada do espaço, fazendo intervir a raiz imaginária de -1, segundo a notação que se tornou padrão ${ }^{3}$ (sem dúvida, ele foi influenciado por uma observação sobre esse assunto feita por Lagrange e utilizada por d'Alembert, cf. Paty, 1998).

Einstein, por sua vez, seguiu (por muitos anos, como atestam os documentos da época, tais como cartas, manuscritos e depoimentos contemporâneos, cf. Paty, 1993, cap. 2, 3) uma reflexão acerca dos problemas do eletromagnetismo que se apoiava na primeira teoria de Lorentz, abordando-os sob o ângulo dos princípios teóricos que

2 Aliás, Poincaré examinou de maneira crítica os conceitos de simultaneidade e de tempo, mas independentemente de seu trabalho sobre a eletrodinâmica, no qual ele não se estende sobre a significação física do conceito, que é tratado matematicamente em suas fórmulas (cf. Paty, 1992, 1996).

$3 \mathrm{x}^{4}=\mathrm{c}$ “-1. A invariante espaço-temporal s é escrita, então, de maneira simétrica pelas quatro coordenadas: $\mathrm{s}^{2}=$ $\mathrm{x}_{1}^{2}+\mathrm{x}_{2}^{2}+\mathrm{x}_{3}^{2}+\mathrm{x}_{4}^{2}$. 
deveriam governar as teorias em jogo, especialmente a questão da relatividade do movimento quando consideramos também os corpos materiais e o éter eletromagnético e óptico. Seu artigo de 1905, "Sobre a eletrodinâmica dos corpos em movimento", representa o resultado desses anos de reflexão ao longo dos quais ele não tinha publicado nada sobre o assunto (cf. Einstein, 1905). A estrutura fundamental da termodinâmica, alicerçada nesses dois princípios, serviu-lhe de guia. Portanto, ele escolheu, por razões físicas muito precisas e argumentadas, o "princípio de relatividade", que a mecânica por sua parte já respeitava, anunciando a necessidade de tornar invariante com relação aos estados de movimento de inércia a teoria eletromagnética de Maxwell reformulada por Lorentz. Em contrapartida, ele exigia da mecânica a submissão a uma característica que a teoria eletromagnética impunha e que, de alguma maneira, a resumia, a saber, que a velocidade da luz no vazio é constante e independente do movimento da fonte de luz, característica a partir da qual ele obtinha um segundo princípio geral da natureza, que os fenômenos físicos deviam respeitar. Os dois princípios pareciam inicialmente contraditórios entre si; com efeito, seguindo as leis do movimento admitidas até então, aquelas da mecânica, a velocidade da luz não poderia ser a mesma nos dois sistemas de referência em movimento relativo, contrariamente à exigência do princípio de relatividade (pois isso significaria que a teoria das ondas eletromagnéticas de Maxwell não é a mesma nos dois sistemas em movimento relativo).

Para conciliar esses dois princípios físicos, ele percebeu que precisava modificar a lei clássica (galileana) da adição das velocidades, que impedia que a velocidade da luz possa ser a mesma em dois sistemas de coordenadas em movimento um em relação ao outro. Isso o conduziu para uma redefinição física das coordenadas do espaço e do tempo, consideradas pelos sistemas de referência respectivamente em repouso ou em movimento; elas deveriam ser submetidas aos dois princípios físicos que acabamos de referir. A modificação da cinemática foi o resultado e Einstein obteve, por dedução dos dois princípios, as fórmulas de transformação das coordenadas do espaço e do tempo que conhecemos como as "fórmulas de transformação de Lorentz", propostas ao mesmo tempo e independentemente, por razões distintas como o vimos, por Lorentz e Poincaré. Resultou disso a reformulação relativista das equações de Maxwell-Lorentz e, portanto, das leis do eletromagnetismo.

Faltaria entrar mais nos detalhes para melhor compreender o alcance e a natureza exata de cada uma das contribuições fundamentais que não fizemos até aqui senão resumir. Não posso mais do que remeter aos estudos mais detalhados já mencionados (no que se refere a minhas próprias pesquisas), assim como às outras descrições, análises e comentários desses trabalhos, mesmo se eles privilegiarem um ou outro ponto de vista, desde que sejam conduzidos pela preocupação de fidelidade aos textos e aos 
fatos. ${ }^{4}$ Não se pode dizer o mesmo de várias publicações recentes que parecem emanar de um grupo, ditadas por a priori ideológicos inconfessáveis, 5 onde o partido tomado no processo de intenção e de calúnia da memória de Einstein é ilustrado por argumentos de enfadonha mediocridade; esses esfregões panfletários apresentam-se tanto sob o gênero da divulgação, mas distorcida e adulterada, como sob o gênero da história da ciência, mas toda ela simplesmente usurpada, como ainda da própria ciência, mas visivelmente mal assimilada e com uma ignorância completa daquilo que pode ser o espírito da pesquisa. Os autores desses péssimos libelos têm em comum, além de uma "einstenfobia" que lembra outros tempos, uma arrogância na sua pretensão de recriar a história e assentar sua pretendida "verdade", em absoluta oposição à inteligência, à modéstia e à generosidade, que são frequentemente o feito dos verdadeiros cientistas e que são seguramente o que é apropriado, de maneira eminente, aos grandes espíritos dos quais falamos neste texto, Lorentz, Poincaré e Einstein.

Vemos, ao contrário, ao ler e ao analisar estes últimos autores, considerando os textos e os documentos na sua historicidade e no seu conteúdo de sentidos, como cada uma das contribuições à teoria da eletrodinâmica ou da relatividade, de Lorentz, de Poincaré e de Einstein, foi inventiva e criativa, seguindo um caminho ditado por uma exigência de compreensão racional própria a cada um deles, e não por uma pretendida “via que seria necessário seguir”, pois não existe uma que seja dada com anterioridade (nem nesse caso, nem em outros). Além do que é dado de fato pelo conhecimento, não existia (e nem existe jamais) senão o desconhecido; não existe via totalmente traçada, nem método seguro, para avançar em tais caminhos; e é por isso que devemos falar de criação. Cada um deles não poderia seguir senão as suas próprias exigências de inteligibilidade racional, que não são as mesmas de um pensador para outro, mesmo entre os melhores especialistas no domínio, pois elas são tributárias de todo tipo de fatores, mas, antes de tudo, porque se trata da representação simbólica e conceitual, diferente em natureza da realidade do próprio mundo.

Vemos bem a diferença e a independência das maneiras de raciocinar de Poincaré e de Einstein em seus trabalhos praticamente simultâneos de 1905, que conduziram às duas teoria das quais as consequências são aproximadamente equivalentes (quanto às

4 Como é o caso de Wolfgang Pauli, Edmund Whittaker, Gerald Holton, Arthur Miller, Abraham Pais, John Stachel, Elie Zahar e de alguns outros. Não posso sobrecarregar o presente artigo dando todas as referências, que podem ser encontradas consultando meus artigos e as obras mencionadas.

5 Pode-se perceber nesses panfletos “denunciadores", tanto pelo seu estilo quanto pelo seu conteúdo, um nacionalismo de exclusão, francês ou mesmo sérvio (neste caso, associado a um feminismo caricatural, que atribui à primeira esposa de Einstein a invenção da relatividade restrita...), anti-semitismo, teorias da conspiração... $\mathrm{Ne}$ nhuma dessas publicações, feitas de citações truncadas e fora de contexto, justapostas sem rigor, e de supostas intenções dadas por provas, responde às mínimas exigências de rigor e de objetividade de uma história da ciência, mesmo que rudimentar. 
fórmulas), mas cuja estrutura e significação conceitual, do ponto de vista físico, são diferentes. Em particular, Poincaré não detalha, na sua teoria, a implicação, para a definição de tempo e de espaço, da nova lei de adição das velocidades, a qual resulta, nele, de uma hipótese empírica (a contração de Lorentz e as transformações que a generalizam). Certamente, ele estaria à altura de fazê-lo, se tivesse percebido a importância, pois ele teria efetuado, em um trabalho independente, uma análise da simultaneidade do tempo em termos de correspondência de sinais. O fato é que ele não considerou essa crítica conceitual em seu trabalho sobre a eletrodinâmica, precisamente porque, sem dúvida, ele estava, sobretudo, voltado para suas preocupações com a dinâmica. Mas essa crítica foi, para Einstein, a solução necessária para o problema posto: a ideia de regular a redefinição física do espaço e do tempo com base nos dois princípios escolhidos, e de perceber que a dinâmica devia ser regulada inicialmente pela cinemática, pertence-lhe por direito.

Algum tempo mais tarde, admitindo totalmente as novas propriedades espaçotemporais requeridas pela "nova dinâmica" (eletromagnética), Poincaré exprimirá a ideia de que se pode manter ao lado delas uma mecânica inalterada em seus conceitos e em suas equações. Para Einstein, ao contrário, a mecânica e o eletromagnetismo clássicos, a primeira sendo reformulada face ao outro, obrigam a uma unificação de seus conceitos. Essa modificação brutal e forçada de concepções bem admitidas e estabelecidas sobre uma ciência de grande solidez como a mecânica poderia, aliás, aparecer então como inutilmente revolucionária.

Tendo resolvido desse modo a questão da originalidade e da diferença das abordagens de Einstein e de Poincaré, podemos considerar a questão de suas justificações. Uma das teorias, no contexto científico dado, é melhor do que a outra? E, quanto a seus respectivos autores, um teria mais razão do que o outro? É evidentemente claro que nenhuma das duas teorias era falsa, mesmo uma em relação à outra. As duas correspondiam a vias de abordagem teóricas possíveis, tanto racionalmente quanto em relação aos dados da experiência. Uma outra maneira de questionar-se seria a de indagar se uma das duas teorias era mais "normal" que a outra. Seria necessário, contudo, definir o que entendemos como "normal" e situar a normalidade em relação ao espírito criativo, do qual os dois trabalhos examinados são testemunhos exemplares; tal exercício seria puramente acadêmico e a própria ideia de normalidade pareceria bem relativa...

Seria desprovido de sentido pretender que Einstein teria feito um erro em relação a Poincaré, por exemplo, ao não atribuir um valor particular à experiência de Michelson e Morley, e ao não ver aí um índice a mais para a relatividade dos movimentos na óptica; ou, ainda, ao fazer uma ideia mais direta da adequação dos conceitos físicos em relação aos fenômenos e ao mundo real. Seria totalmente arbitrário julgar o trabalho de Poincaré pelo padrão daquele de Einstein e criticá-lo por não ter feito 
avançar os dois princípios, e de não tê-los formulado senão como uma consequência da invariância com relação às fórmulas de transformação de Lorentz, escolhidas empiricamente por sua propriedade notável na relação com termos das diferentes ordens de movimento. As posições de um e de outro revelam escolhas possíveis, perfeitamente legítimas.

Ninguém, nessa época, poderia dizer que a teoria de um era melhor que a do outro. Se a eletrodinâmica fosse considerada, como pensavam muitos físicos de então, o fundamento da física em seu conjunto, a teoria de Poincaré, que era uma eletrodinâmica relativista, teria talvez sido a abordagem doravante privilegiada. Entretanto, nem mesmo isso é seguro, pois Paul Langevin, próximo de Poincaré e ele mesmo partidário de uma "visão eletromagnética do mundo", entendeu que a teoria de Einstein era mais direta e de alcance mais geral e tornou-se o propagandista dessa teoria na França, mantendo ainda durante alguns anos seu pensamento do "todo eletromagnético" e uma referência ao éter (cf. Paty, 2002). De fato, antes da teoria da relatividade geral, cada uma das teorias, tanto a de Poincaré como a de Einstein, tinha seus partidários. Os matemáticos aprovavam mais a teoria de Poincaré, mais formalizada e mais sintética no que concerne às implicações dos grupos de transformação (cf. Walter, 1996). ${ }^{6}$ Os físicos que em geral ignoravam a teoria de Poincaré, publicada em uma revista de matemática, eram eventualmente mais sensíveis à argumentação diretamente física de Einstein sobre a generalização de um princípio de fato constatado da relatividade do movimento na mecânica, na óptica e no eletromagnetismo, e à coerência da reforma fundamental do espaço e do tempo por colocar esses conceitos em harmonia com a rejeição das ações instantâneas a distância e pela plena aceitação do conceito de campo. (Quando ao menos não rejeitaram essa reforma, julgaram-na por mui-to tempo inutilmente revolucionária e iconoclasta, em particular os mecânicos, cf. Paty, 1987.)

Mas na época da publicação desses trabalhos, não constatamos nenhum consenso entre os cientistas do domínio (físicos ou matemáticos), cujas atitudes com relação a esses resultados poderiam ser muito variadas. O físico Lorentz rejeita inicialmente toda a abordagem de Einstein, apesar de sua estima por ele, preferindo a teoria de Poincaré, mais próxima de sua visão (cf. Lorentz, 1921). O matemático Hermann Minkowski adota a física de Einstein casando-a com o formalismo de Poincaré, estabelecendo, assim, a teoria físico-matemática do espaço-tempo (cf. Minkowski, 19o7, 1908, 1909).

6 Tal foi ainda, muitos anos mais tarde, a escolha de Edmund Whittaker que, contrariamente à tendência geral da época, em 1953, atribui a teoria da relatividade restrita a Poincaré, sem dizer uma palavra da contribuição de Einstein. Por outro lado, Whittaker atribui com justiça apenas a Einstein a descoberta da teoria da relatividade geral (cf. Whittaker, 1953). 
Mais tarde, efetuou-se uma escolha entre as duas teorias, privilegiando a via tomada por Einstein; isso aconteceu no momento do sucesso da teoria da relatividade geral, suscitada especialmente pela constatação astronômica de uma curvatura do espaço nas proximidades de grandes massas (durante o eclipse solar de 1919, graças à observação do deslocamento da posição no céu das estrelas vizinhas ao disco solar, relacionada a uma inflexão dos trajetos luminosos). Esta teoria expandia a da relatividade restrita ao situar-se, do ponto de vista da física, na mesma perspectiva de princípio que aquela (a saber, que a teoria está fundamentada sobre princípios físicos de alcance universal) e reformulou novamente o espaço e o tempo, ligando-os às massas materiais, fontes dos campos de gravitação. Ela apresentava, além de seu valor quanto à explicação e à predição dos fenômenos da natureza, uma perfeição matemática que Poincaré não tinha certamente alcançado e que foi, aliás, reconhecida imediatamente pelos matemáticos, de David Hilbert a Elie Cartan.

Foram, por outro lado, os matemáticos que desenvolveram em seguida a teoria da relatividade geral que os físicos abandonaram por muito tempo, passado o primeiro entusiasmo, por não dispor, então, de mais do que três testes experimentais ou observacionais. Mas a teoria da relatividade restrita, que tinha sido adotada no entusiasmo causado pela teoria da relatividade geral, sobreviveu ao esquecimento provisório desta última, vindo a tornar-se um dos principais instrumentos de pensamento nos novos domínios da física que se abriam. Adotando a relatividade restrita, eles adotaram também a direção dos princípios e dos conceitos considerados por Einstein; ela excedeu sem dificuldades o objetivo específico da eletrodinâmica clássica e se impôs como modelo para os conceitos de espaço, de tempo, de causalidade espaço-temporal e de energia, que estavam em jogo. Nesse contexto, a contribuição, apesar de historicamente fundamental, de Poincaré foi largamente esquecida, entenda-se, subavaliada. Como indicamos, restam, entretanto, na recepção e na compreensão posteriores, até os nossos dias, da relatividade restrita einsteiniana, elementos importantes que são originariamente de Poincaré (grupos de Lorentz e de Poincaré, o tempo como quarta coordenada, a invariante $\mathrm{ds}^{2}$ etc.). Acerca disso, a denominação usual de "transformação de Lorentz", devida a Poincaré, tem valor de índice.

A ciência julgada, ou sancionada, como a qualificou Gaston Bachelard, esquece frequentemente das circunstâncias que a tornam possível, e é a tarefa da história da ciência fazer-nos reviver os percursos efetivos pelos quais o que sabemos hoje em dia apareceu em um dia e conscientizar-nos, ao mesmo tempo, que antes disso esse saber não existia. Como esse absolutamente novo pôde surgir é uma questão que não abordaremos neste texto. Ficaremos contentes em notar que esse surgimento se efetuou por processos de trabalho de pensamento que têm direito, propriamente falando, ao qualificativo de criação. Dessas criações combinadas e rearranjadas resulta aquilo que 
conhecemos. Os trabalhos paralelos de Lorentz, de Poincaré e de Einstein, que estabeleceram o princípio de relatividade na física (para os movimentos de inércia), ilustram maravilhosamente a diversidade de estilos de pensamento e de pesquisa na criação científica e, também, a maneira complexa, diversificada, apesar das aparências, ${ }^{7}$ pelas quais realmente contribuíram para a formação dos conhecimentos presentes e para a nossa compreensão atual. Basta dizer que não abandonamos, com as nossas questões acerca do estilo e da criação, o terreno do racional.

\section{Pensamento Reflexivo e Filosofia da Griação RaGional}

Podemos, portanto, retornar ao tema filosófico que esboçamos no início, a saber, se podemos falar de maneira racional da "criação científica" e se o processo dessa criação depende ele mesmo da racionalidade. A noção de "estilo científico" 8 permite compreender que não existe uma via racional única para descobrir aquilo que era desconhecido. Um conhecimento novo não está inscrito com anterioridade, em potência, e sob uma única forma possível, em um céu de ideias, mas é fruto de um processo de criação pelo pensamento. Se esse fruto é eminentemente racional, seria surpreendente que os meios de colocá-lo em operação sejam somente irracionais, como pretenderam numerosos filósofos da ciência do século xx. E, na verdade, existe muito de racionalidade nesse processo; ele passa pelas exigências de inteligibilidades particulares, mas o resultado obtido transcende necessariamente essas últimas, e propõe-se imediatamente como objetivo e universal. Comunicados aos outros pensamentos que se aplicam igualmente a esse campo científico, os problemas e resultados científicos, inicialmente formulados por inteligências singulares, ultrapassam essa singularidade da formulação de um só para verem-se assimilados e, eventualmente, modificados para alcançar uma compreensão mais universal. Jean Cavaillès lembra, nesse sentido, a necessidade de uma "filosofia do conceito" para resolver a contradição aparente entre a invocação de um sujeito transcendental, cadinho do trabalho de pensamento, e a da objetividade (racional) das proposições que resultam dessa atividade (cf. Cavaillès, 1976).

${ }_{7}$ É assim que, quando falamos hoje em dia de "teoria de Einstein da relatividade restrita", designamos uma teoria que integra outros aportes, contemporâneos e posteriores, aos artigos de Einstein acerca do assunto. Assim também a mecânica dita newtoniana é constituída de numerosos aportes contemporâneos a Newton (Huygens, Leibniz) ou posteriores a ele (especialmente a formulação em termos do cálculo diferencial e integral).

8 Acerca da noção de estilo científico, remeto especialmente a Gilles G. Granger, que abordou essa questão sob uma perspectiva filosófica, cf. Granger, 1968. Diversos outros autores abordam este tema de maneira simplesmente descritiva, do ponto de vista da história da ciência. 
Como veremos, Poincaré situa-se nesta perspectiva; ele não nos leva apenas a considerar sua obra criativa e a desenrolarmos por nós mesmos o trabalho que ele conduziu; ele propôs, de maneira direta ou indireta, uma reflexão verdadeiramente filosófica sobre o pensamento matemático, físico, científico de uma maneira geral, concebendo-a tanto na dimensão de racionalidade quanto naquela de um pensamento que, pretendendo a inteligibilidade, é conduzido a ultrapassar o que é dado, quer dizer, a inventar, a criar.

O próprio Poincaré indica-nos que seu principal interesse na filosofia dirige-se aos aspectos criadores do pensamento científico. Mais tarde, Einstein declararia ele também uma opção da mesma ordem e, de fato, parece certo que esses dois cientistas e filósofos são os dois principais pensadores dessa época acerca do que podemos chamar o pensamento científico criativo concebido em uma perspectiva racional. 9 Einstein pertence expressamente à mesma linhagem de Poincaré nesse aspecto, ainda que orientasse um pouco diferentemente sua filosofia, que se pode qualificar de "realismo crítico", para diferenciá-lo do "convencionalismo" de Poincaré. Os dois baseiam suas considerações na "liberdade lógica" ou "livre escolha lógica" entre os fatos e as proposições teóricas, a qual expressa uma constatação retirada da crítica à indução de David Hume (a qual era também, em certo sentido, a posição de Ernest Mach e de Pierre Duhem). Tanto Poincaré como Einstein tiveram simultaneamente a ideia de criação científica e a de sua liberdade, a racionalidade e a inteligibilidade, a intuição e a invenção.

Essas teses não eram, então, evidentes (hoje em dia, elas são ainda muito pouco tomadas em consideração) em razão do predomínio do neopositivismo e do empirismo lógico ou, ao contrário, das perspectivas antirracionalistas da época de Einstein; e, quanto à época de Poincaré, em razão do predomínio do psicologismo e do pragmatismo, ou do espiritualismo ou, ainda, de uma concepção estreita do determinismo. De fato, uma condição para que a ideia de criação concernente ao conhecimento científico possa ser levada em consideração é a conscientização, relativa a esse conhecimento, de sua natureza de representação simbólica, distinta do objeto representado. Essa distanciação entre a representação conceitual e simbólica e seu objeto aconteceu no século xıx, com as reconstruções das teorias matemáticas, na aritmética e na álgebra, com a descoberta, na geometria, das geometrias não-euclidianas e com a formalização matemática cada vez mais acentuada da física e sua elaboração de conceitos mais abstratos, não diretamente legíveis nos fenômenos da natureza (por exemplo, a formulação hamiltoniana

9 Outros filósofos, especialmente Henri Bergson e William James, debruçaram-se sobre a questão da criação, artística ou científica, pelo pensamento, mas de uma perspectiva empirista ou pragmática, ou ainda psicológica, privilegiando uma sensibilidade não racional (cf. Bergson, 1934; James, 1911). 
da mecânica, a elaboração da termodinâmica com os conceitos de estado e de entropia, mais tarde, o conceito de campo desligado do suporte do éter etc.). Aparecia visivelmente que as teorias científicas resultam de um trabalho de elaboração e de construção, pelo pensamento, sobre os objetos (simbólicos) do pensamento. Mencionemos igualmente, sem insistir, que uma tomada de consciência análoga ocorreu nas artes, culminando com o movimento simbolista e resumida na expressão de Mallarmé para designar a pintura de uma rosa em um quadro, marcando a sua irremediável diferença com a rosa real, "a ausência completa de buquê".

Esse caráter simbólico e construído do conhecimento indica muito bem as limitações de uma "filosofia da natureza", para a qual o conhecimento científico é concebido segundo uma continuidade com o mundo.

Faltaria aqui tratar com detalhes dos elementos da filosofia do conhecimento de Poincaré, para fazer ver as ligações entre eles e sua própria coerência. Aqui não posso mais do que indicar o início de um tal estudo. Podemos marcar esse início lembrando uma frase de Poincaré que pode parecer à primeira vista anódina, mas plena de significação quanto à consciência do caráter da representação mental, simbólica, do conhecimento em relação à realidade da natureza e de sua distinção com ela.

Essa frase é a seguinte: "o cérebro humano, que é um pequeníssimo pedaço do universo, não poderia conter esse universo" (Poincaré, 1908a, p. 20). Dito de outra maneira, o pensamento é devotado, por seu próprio pertencimento à natureza e sua inserção nela (que nos lembra, aliás, a teoria da evolução e, em nossos dias, a cosmologia contemporânea), a constituir, pelo seu próprio uso e com seus os próprios meios, uma representação da realidade do mundo que não pode, por definição, coincidir com esse mundo, ao mesmo tempo em que se propõe ser dele uma imagem fiel (segundo as capacidades do entendimento, as exigências da inteligibilidade). A fidelidade dessa imagem será, de fato, aquela das relações entre esses elementos em vista daquelas que podemos atribuir ao mundo segundo uma correspondência "analógica" (o termo analogia é tomado neste texto no sentido de analogia estrutural "matemática", desenvolvida em outros contextos e várias vezes retomada por Poincaré). O pensamento humano está, portanto, posto na situação de criar, ou seja, de forjar com todas as peças, com seus próprios materiais, mentais e simbólicos, com suas categorias de pensamento e seus conceitos, sua representação do mundo, isto é, o conhecimento que ele obtém deste mundo. Certamente, ele o fará no mundo, do qual ele é parte constituinte, mas também contra ele, porque ele precisa escolher, dentre os fatos que serão as garantias da correspondência e da adequação, aqueles que são significativos e ignorar ou deixar de lado os outros, que não produzem novas indicações e que são muitos (cf. Poincaré, 1908d). A descrição é inversa à do cartógrafo excessivamente meticuloso do conto de 
Jorge Luis Borges, que, com sua preocupação de exatidão, procurava desenhar seu mapa em exata coincidência com o modelo real...

Nesse trabalho de criação de uma representação, o papel mais fundamental é o da intuição, pela qual Poincaré reconheceu um caminho sintético global e imediato, que precedeu todo o trabalho de análise efetuado em seguida para assegurar a certeza. Trata-se de uma função do intelecto, isto é, do entendimento, que opera na racionalidade. Para Poincaré, no que diz respeito às matemáticas, a intuição que inventa (que tem a função da invenção, no pensamento dos matemáticos intuicionistas, mas também naquele dos analistas, que consideram primeiramente o aspecto lógico, pois eles também têm suas formas de intuição, cf. Poincaré, 1889, 1905a) é uma função propriamente racional; dito de outra maneira, ela é uma intuição racional, capaz de inventar racionalmente formas racionais, correspondentes de uma maneira particularmente adequada às formas do mundo real e que constituem o conhecimento científico. Essa consideração é plena de implicações para a natureza da invenção científica e para a natureza do racional que torna essa invenção possível; e isso é tudo o que gostaria de dizer neste texto.

Agradecimentos. Este trabalho foi preliminarmente apresentado no Colloque Henri Poincaré "Science et Pensée", em mesa redonda ocorrida em 17/jan./2005, em Sophia-Antipolis.

Traduzido do original em francês por Claudemir Roque Tossato e Pablo Rubén Mariconda.

Michel PATY

Diretor de pesquisa emérito do Centre National de la Recherche Scientifique. Equipe REHSEIS e Universidade de Paris 7, França. michel.paty@univ-paris-diderot.fr

\begin{abstract}
We try, in this article, to enlighten Henri Poincaré's "style" in theoretical physics and mathematics, by analyzing in particular the case of his theoretical formulation of relativistic electrodynamics, and the place occupied in it by the principle of relativity. A comparison of this creative work with the parallel one done at the same time by Einstein (of the special relativity) allows to put in evidence the contrast between these two approaches. We evoke then the novelties that Poincaré's work and thought bring into the domain of philosophy concerning the problem of scientific creation and its relation to rationality.
\end{abstract}

KEYwords $\bullet$ Scientific creation. Intuition. Invention. Rationality. Principle of relativity.

Electrodynamics. Theoretical physics. Mathematics. 


\section{REFERÊNGIAS BIBLIOGRÁFICAS}

Bergson, H. La pensée et le mouvant. Paris: Alcan, 1934.

Boi, L.; Flament, D. \& Salanski, J. M. (Ed.). 1830-1930: a century of geometry, epistemology, history and mathematics. Berlin: Springer-Verlag, 1992.

Cavaillès, J. Sur la logique et la théorie de la science. Paris: Vrin, 1976.

Einstein, A. Elektrodynamik bewegter Körper. Annalen der Physik, 4, 17, p. 891-921, 1905.

Flament, D. (Ed.). Dimensions, dimensions I. Paris: Fondation Maison des Sciences de l'Homme, 1998.

GLIck, T. (Ed.) The comparative reception of relativity. Dordrecht: Reidel, 1987 .

Granger, G. G. Essai d'une philosophie du style. Paris: Armand Colin, 1968.

Greffe, J. L.; Heinzmann, G. \& Kuno, L. (Ed.). Henry Poincaré: science et philosophie. Science and philosophy. Wissenschaft und Philosophie. Paris/Berlin: Albert Blanchard/Akademic Verlag, 1996.

Hadamard, J. An essay on the psychology of invention in the mathematical field. Princeton: Princeton University Press, 1945 .

James, W. Le pragmatisme. Paris: Flammarion, 1911.

LoRenTz, H. Versuch einen Theorie der elektrischen und optiken Erscheinungen in bewegten Körpern. Leiden: Brill, 1895 .

. Electromagnetic phenomena in a system moving with any velocity smaller than of light. Proceedings of the section on science, 6, p. 809-31, 1904.

Deux mémoires de Henri Poincaré sur la physique mathématique. Acta Mathematica, 38, p. 293 $3 \circ 8,1921$.

Mascart, E. Sur les modifications qu'éprouve la lumière par suite du mouvement de la source lumineuse et du mouvement de l'observateur. Annales Scientifiques de l'École Normale Supérieure, 2, 1, p. 157-214, 1872 .

. Sur les modifications qu'éprouve la lumière par suite du mouvement de la source lunineuse et du mouvement de l'observateur. Annales scientifiques de l'Ecole Normale Supérieure, 2, 3, p. 363-420, 1874 .

Maxweld, J. C. A treatise on electricity and magnetism. New York: Dover, 1954 [1873] .

Minkowski, H. Das Relativitätsprinzip. Annalen der Physik, 47, p. 927-38, 1907.

. Die Grundgleichungen für die elektromagnetische Vorgänge in bewegten Körpern. Nachrichten der Königlichen Gesellschaft der Wissenschaft und der Georg-August Universität zu Göttingen, Mathematischphysikalische Klasse, p. 53-111, 1908.

. Raum und Zeit. Physikalische Zeitschrift, 10, p. 104-11, 1909.

Oliveira, M. P. E. Mascart et l'optique des corps en mouvement. Paris, 1993. Tese (Doutorado em Filosofia). Université Paris 7- D. Diderot.

PATY, M. The scientific reception of relativity in France. In: GLICK, T. (Ed.) The comparative reception of relativity. Dordrecht: Reidel, 1987. p. 113-67.

Physical geometry and special relativity: Einstein and Poincaré. In: Boi, L.; Flament, D. \& SaLAnski, J. M. (Ed.). 1830-1930: a century of geometry, epistemology, history and mathematics. Berlin: SpringerVerlag, 1992. p. 126-4.9.

Einstein philosophe. La physique comme pratique philosophique. Paris: PUF, 1993.

Poincaré et le principe de relativité. In: Greffe, J. L.; Heinzmann, G. \& Kuno, L. (Ed.). Henry Poincaré: science et philosophie. Science and philosophy. Wissenschaft und Philosophie. Paris/Berlin: Albert Blanchard/Akademic Verlag, 1996. p. 101-43.

. Les trois dimensions de l'espace et les quatre dimensions de l'espace-temps. In: Flament, D. (Ed.). Dimensions, dimensions I. Paris: Fondation Maison des Sciences de l'Homme, 1998. p. 87-112.

. La place des principes dans la physique mathématique au sens de Poincaré. Fundamenta Philosophiae, 3, 2, p. 61-74, 1999a. 
Paty, M. La création scientifique selon Poincaré et Einstein. In: Serfati, M. (Ed.). La recherche de la vérité. Paris: ACL-Éditions du Kangourou, 1999b. p. 24,1-80. . Poincaré, Langevin et Einstein. Épistémologiques, 1-2, p. 33-73, 2002.

Poincaré, H. Du role de l'intuition et de la logique en mathématiques. Compte-rendus du Deuxième Congrès International des Mathématiciens. Paris: [s.n.], p. 115-30, 1889. . La valeur de la science. Paris: Flammarion, 1905 a. Sur la dynamique de l'électron. Compte-rendus des Séances de l'Académie des Sciences, 14, o, p. 1504$8,1905^{b}$.

. Sur la dynamique de l'électron. Rendiconti del Circolo matematico di Palermo, 21, p. 129-76, 1905c. Science et méthode. Paris: Flammarion, 1908a.

Comment on invente. Le travail de l'inconscient. Le Matin, Paris, 24 Dez. 1908b.

. L'invention mathématique. Bulletin de L'Institut General de Psychologie, 3, p. 175-96, 1908c. Le choix des faits. The Monist, p. 231-2, 1908d.

Popper, K. R. The logic of scientific discovery. London: Hutchinson, 1959.

Objective knowledge, an evolutionary approach. Oxford: Clarendon Press, 1972.

Reichenbach, H. Experience and prediction. Chicago: University of Chicago Press, 1938.

Serfati, M. (Ed.). La recherche de la vérité. Paris: ACL-Éditions du Kangourou, 1999.

WaLter, S. Hermann Minkowski et la mathématisation de la théorie de la relativité, 1905-1915. Paris, 1996.

Tese (Doutorado em Filosofia). Université Paris 7-D. Diderot.

Whittaker, E. T. A history of the theories of aether and electricity. London: Nelson, 1953. v. 2. 